Portland State University

PDXScholar

\title{
The Imposter Phenomenon among Emerging Adults Transitioning into Professional Life: Developing A Grounded Theory
}

Joel A. Lane

Portland State University, lanejoel@pdx.edu

Follow this and additional works at: https://pdxscholar.library.pdx.edu/coun_fac

Part of the Counseling Psychology Commons, and the Student Counseling and Personnel Services Commons

Let us know how access to this document benefits you.

\section{Citation Details}

Lane, Joel A., "The Imposter Phenomenon among Emerging Adults Transitioning into Professional Life: Developing A Grounded Theory" (2014). Counselor Education Faculty Publications and Presentations. 24. https://pdxscholar.library.pdx.edu/coun_fac/24

This Post-Print is brought to you for free and open access. It has been accepted for inclusion in Counselor Education Faculty Publications and Presentations by an authorized administrator of PDXScholar. Please contact us if we can make this document more accessible: pdxscholar@pdx.edu. 
The Imposter Phenomenon among Emerging Adults Transitioning into Professional Life:

Developing A Grounded Theory

Joel A. Lane

Oakland University

Author Note:

Joel A. Lane, Department of Counseling, Oakland University, Rochester, MI.

Joel A. Lane is now at the Department of Counselor Education, Portland State University, Portland, OR.

Correspondence concerning this article should be addressed to Joel A. Lane, Department of Counselor Education, Portland State University, Graduate School of Education, P.O. Box 751, 615 SW Harrison, Room 504A, Portland, OR 97207. (e-mail: lanejoel@pdx.edu).

The author wishes to acknowledge the help of Dr. Stephanie Crockett, who served as an auditor during the data analysis and provided comments on a previous draft of this manuscript, and Carol Burns-Wortham, who also served as an auditor. 


\begin{abstract}
This study qualitatively explored the imposter phenomenon among 29 emerging adults who were transitioning into professional life. A grounded theory was developed that described the imposter phenomenon, internal and external contributing factors, and its impact in terms of performance and affective reactions. Implications for counselors of emerging adults are discussed.

Keywords: emerging adulthood, imposter phenomenon, transition
\end{abstract}


The Imposter Phenomenon among Emerging Adults Transitioning into Professional Life:

Developing A Grounded Theory

The developmental trajectories of young adults between the ages of 18 and 25 have undergone considerable change over the past several decades (Arnett, 2000). Today's emerging adults tend to identify with aspects of both adolescence and adulthood without fully identifying with either (Arnett, 2001). This lack of clear identification can create identity tension as emerging adults transition from adolescent to adult life roles, generally in some form of career and/or higher education (Murphy, Blustein, Bohlig, \& Platt, 2010). This identity tension poses a threat to the process of one's initial transition into professional life, a transition associated with feelings of low self-efficacy and turmoil (Polach, 2004).

These feelings bring to mind the imposter phenomenon (IP; Clance \& Imes, 1978), a construct that has received considerable attention in higher education and psychological research (e.g., Bernard, Dollinger, \& Ramaniah, 2002; Cowman \& Ferrari, 2002; Gibson-Beverly \& Schwartz, 2008; Royse-Roskowski, 2010; Sonnak \& Towell, 2001). Given that emerging often adults enter career life - a role commonly associated with adulthood - despite subjectively identifying as somewhere between adolescence and adulthood (Arnett, 2000), it seems possible that the IP is a salient construct for this transition. Thus, the present study examined the experience of the IP among emerging adults transitioning into professional life.

\section{The Imposter Phenomenon}

The IP refers to a feeling of incompetence despite evidence of competence (Clance \& Imes, 1978). Individuals experiencing the IP see themselves as less capable than their peers and have difficulty internalizing successes (Leary, Patton, Orlando, \& Funk, 2000). Moreover, the IP promotes feelings of fraudulence, causing individuals to perceive that their abilities are 
overestimated and that others will eventually discover their incompetence (Clance \& Imes, 1978). High-achieving individuals seem especially susceptible to the IP (Clance \& Imes, 1978; Fried-Buchalter, 1997; Gibson-Beverly \& Schwartz, 2008). While imposter feelings can lead some to work harder and over-prepare (Caselman, Self, \& Self, 2006), it also can also promote self-handicapping behaviors such as procrastination or lack of effort (Cowman \& Ferrari, 2002; Want \& Kleitman, 2006). Common IP correlates include anxiety (Topping, 1983), depression (McGregor, Gee, \& Posey, 2008), low self-esteem (Sonnak \& Towell, 2001), low self-efficacy (Royse-Roskowski, 2010), high neuroticism and low conscientiousness (Bernard et al., 2002), narcissism (Gibson-Beverly \& Schwartz, 2008), and proneness to shame (Cowman \& Ferrari, 2002). Essentially, the IP poses a significant threat to well-being (Clance \& O'Toole, 1988).

\section{The Transition to Career}

Several of these psychological challenges - especially anxiety and low self-efficacy mirror those experienced when individuals transition into career settings (Cherniss, 1980; Polach, 2004). The challenges associated with this transition are well-documented, and they are frequently used to explain growing job turnover rates among new professionals (Saks, Uggerslev, \& Fassina, 2007). New professionals report numerous sources of confusion while entering the workplace, including unanticipated changes in structure, oversight, and appropriateness regarding interpersonal interactions (Polach, 2004). A study involving a sample of librarians found associations between complex job duties and elevated imposter feelings, especially among those for whom the complex duties were new (Clark, Vardeman, \& Barba, in

press). Compounding these challenges, entry into the workforce often coincides with decreased contact with peer and family support networks (Lane, 2013, in press). Perceiving diminished interpersonal support can stimulate feelings of isolation and exacerbate the psychological 
difficulties associated with this transition (Lane, 2014; Murphy et al., 2010).

Despite these difficulties, relatively little research exists examining the IP in the transition to career. Comparatively, recent research pertaining to new professionals has more commonly focused on the related construct of self-efficacy (i.e., one's beliefs regarding his/her abilities; e.g., Al-Darmaki, 2004). Low self-efficacy is a normative experience for new professionals lacking in workplace experience, and it is relatively independent of actual job performance (Judge, Jackson, Shaw, Scott, \& Rich, 2007). Though similarities exist between the constructs of the IP and low self-efficacy, so too do conceptual and empirical distinctions. Conceptually, an individual low in self-efficacy might feel incompetent, but an individual experiencing the IP would feel incompetent despite evidence of competence (Leary et al., 2000). Empirically, the antecedent construct of high-achievement orientation is a risk factor for the IP (Gibson-Beverly \& Schwartz, 2008), and yet it is positively correlated with self-efficacy (Bell \& Kozlowski, 2002). Moreover, Royse-Roskowski (2010) provided important empirical support for this distinction by examining a sample of counseling trainees, finding that previous work experience improved self-efficacy but not imposter feelings. Essentially, the IP seemed more resistant to prior experience than did self-efficacy. These findings support the distinction between the two constructs and further underscore the need for a better understanding of the IP among young professionals.

\section{The Imposter Phenomenon in Emerging Adulthood}

Accordingly, the IP could represent a problem of particular relevance to emerging adults, as one's initial exposure to meaningful career activities most commonly occurs during this life period (Arnett, 2000). Emerging adults leave behind the moratorium from responsibilities that adolescence represents to enter adult roles (Lane, 2013). In doing so, they enter unfamiliar 
environments with seemingly vague expectations surrounded by more experienced colleagues (Polach, 2004). Today's emerging adults also characteristically engage in a high degree of selfmonitoring (Schnyders \& Lane, 2014), a behavior that is considered developmentally normative in this age group (Arnett, 2000) but is also predictive of imposter feelings (Kolligian \& Sternberg, 1991). Similarly, the aforementioned association of the IP with narcissism (GibsonBeverly \& Schwartz, 2008) supports its relevance in this period of life, as some evidence points to increasingly narcissistic attitudes among present-day emerging adults (Twenge, 2013). Moreover, a risk factor for the IP is having experienced high parental control (Sonnak \& Towell, 2001), which further supports the IP's relevance in emerging adulthood given what some have identified as a growing societal trend toward parental over-involvement continuing well past adolescence (Padilla-Walker \& Nelson, 2012; Twenge, 2013). Though, intuitively, the IP seems like a problem highly relevant to emerging adulthood, it is difficult to make this conclusion given its lack of empirical attention. The most pertinent existing research in this regard involves undergraduate academic settings (e.g., Kolligian \& Sternberg, 1991; Sonnak \& Towell, 2001) and, therefore, has limited applicability to emerging adults entering work life.

Counseling professionals who work with emerging adults in career, college, or mental health settings would benefit from a richer understanding of IP experiences in emerging adults transitioning into the workforce. Given the aforementioned associations between the IP and numerous negative mental health characteristics, it seems likely that IP experiences are a relatively common issue among client populations. The IP, however, constitutes an internal experience easily overlooked in therapy (Clance \& Imes, 1978). As such, an increased understanding of the IP would benefit counselors working with emerging adult populations, as this life phase represents a likely risk factor for elevated imposter feelings. Such an 
understanding could assist counselors in promoting self-efficacy for their emerging adult clients.

\section{Present Study}

In light of its relevance to counselors, the topic of the IP in emerging adult professionals seems well suited for qualitative methodology. Qualitative research allows for rich understandings of behaviors and affect (Denzin, 2006), which seems particularly useful for counselors given the relative commonality with which the IP is overlooked. Despite the apparent utility of this methodology, existing IP research has almost exclusively employed quantitative methodology, specifically correlational designs. Though such an approach has yielded helpful contributions, incorporating additional methodological approaches would enrich our understanding of the IP.

Thus, the present study qualitatively explored the experience of the IP among emerging adults transitioning into professional life. The purpose of the study was to develop a grounded theory (Corbin \& Strauss, 2008) to understand predictive factors of the IP and its impact on emerging adult occupational or academic functioning. The following question guided each element of the present study: Is the IP a relevant construct to emerging adults entering professional life, and if so, how is this construct experienced?

\section{Method}

The present study utilized an emergent grounded theory design. Grounded theory provided an ideal approach since it affords a systematic method of generating theoretical understandings regarding aspects of the social world (Corbin \& Strauss, 2008). This approach requires multiple stages of data collection and analysis (Creswell, 2009). The present study was designed in accordance with this requirement: participants first completed exploratory qualitative surveys, and then a subsample of the participants gave follow-up, in-depth individual interviews. 
Both waves of data were analyzed together to arrive at an emerging model of the IP grounded in the views of the participants (Corbin \& Strauss, 2008).

An important aspect of ensuring the integrity of qualitative procedures involves considering inherent biases the researcher may have regarding the study topic (Kline, 2008). Thus, prior to the present procedure development and participant recruitment, the researcher engaged in personal and collegial reflection to consider his prior experiences and assumptions. Several pertinent experiences were identified. First, the researcher's clinical specialty involves adolescent and emerging adult populations. Second, as an educator, the researcher has been exposed to many emerging adult students and has observed IP experiences in both students and colleagues. Third, the researcher considered his age and its relative proximity to the emerging adult age range. Through reflection with colleagues, the researcher was encouraged to view these experiences as both risks and benefits to the present study. Clearly, the risks involved potential biases and assumptions that could emerge as a result of such close proximity to the topic. The benefits, however, were that such experiences could shape the researcher's expertise with the topic, contribute to establishing rapport with participants, and contribute to relevant protocol development (Kline, 2008). To minimize these risks, it was agreed upon that all aspects of protocol development and data analysis would utilize close oversight from numerous colleagues of various ages and experience levels (Corbin \& Strauss, 2008). In each phase of the study's development, the researcher would draft the element of the study (e.g., survey and interview protocols) and would seek feedback from at least two trusted colleagues, who would comment on the potential efficacy and bias of each element. Such an approach was utilized for the development of protocols, codes, concepts, and categories (Corbin \& Strauss, 2008).

\section{Procedures and Protocols}


The present study utilized two phases of data collection. The first phase involved exploratory qualitative surveys. Individuals who completed the surveys were invited to participate in follow-up interviews, which constituted the second phase of the data collection.

In order to facilitate discussion regarding the IP, a term with which many individuals are unfamiliar (Clark et al., in press), and a construct that represents a private, internal, emotional experience (Clance \& Imes, 1978), both data collection methods utilized a brief reading passage. Precedence exists for utilizing reading passages in qualitative studies with young adult populations (Allen \& Taylor, 2006; Kruger et al., 2013). The passage contained a fictional narrative of an individual experiencing the IP in a professional setting. Several of the initial IPrelated questions in each data collection method pertained to the narrative. Utilizing the narrative offered the semantic advantage of defining and normalizing the IP in the hopes that participants would feel more comfortable discussing their own potential insecurities and feelings of inadequacy. Steps were taken in both the data collection and analysis procedures to minimize the potential for undue influence of the reading passage on the results. Anytime the participants answered a question specifically related to the character in the narrative, they were then asked to compare that answer with their own experiences. Additionally, while analyzing the data, responses that solely pertained to the narrative were not coded.

The survey was electronic and consisted of 19 questions. The questions were generated using previous IP research as well as the researcher's prior clinical and educational experiences (Kline, 2008). Participants were asked to describe their professional or academic identities (e.g., "Please describe what you consider your primary profession to be") and compare their experiences with those described in the narrative (e.g., "When you were reading this passage, could you relate to some of the things that Julie was experiencing? If 'yes', please describe your 
own similar experiences. If 'no', describe how Julie's experiences differ from your own”). Participants were entered in a prize drawing for a $\$ 50$ gift card.

The researcher conducted the follow-up interviews, which took place in a private room in a University counseling center. The interviews lasted 30-55 minutes and contained 22 questions and probes. Participants were asked to read the passage and respond to questions seeking to understand both the degree to which they related to the IP, and also to probe for their own potential imposter experiences (e.g., "Now that we've read and talked a little about the IP, how do some of these concepts resonate with your own personal journey, if they do at all?").

\section{Participants}

The participants were 18- to 25-year-olds who were either not enrolled in an educational program or who were within one year of graduating from their respective programs. The rationale for such parameters was that these emerging adults were likely to be participating in a profession or preparing to transition into professional life by engaging in activities such as job searches, résumé development, career decision-making, etc. (Arnett, 2000). Participants were recruited through various means in connection with a medium-sized, suburban Midwestern University. Recruitment messages were sent through various University mailing lists that reach alumni, entry-level university professionals, and current undergraduate and graduate students. Additional participants were identified through recruitment messages given during class sessions for several undergraduate and graduate courses.

Initial participation in the study consisted of 29 emerging adults completing qualitative surveys. This sample was predominantly female $(n=20,68.9 \%)$ and Caucasian $(n=27,93.1 \%)$. The majority of participants had earned a bachelors degree $(n=16,55.2 \%)$, while others had earned a masters degree $(n=4,13,8 \%)$ or were finishing an undergraduate or graduate program 
$(n=9,31.0 \%)$. The sample represented many professional and academic fields, including business administration, education, student affairs administration, music, engineering, psychology, and counseling.

Each survey participant was invited to participant in a follow-up interview. Six total interviews were conducted. The majority of the interviewees were female $(n=5,83.3 \%)$ and white $(n=4,66.7 \%)$. Two participants had undergraduate degrees and were working in related fields, one participant had an undergraduate degree and was working at a restaurant while looking for a permanent career, one participant had a master's degree and was working part time in a related field, and the other two participants were current students.

\section{Data Analysis}

The researcher transcribed all individual interviews and closely reviewed all submitted surveys. The interviews were analyzed using an inductive microanalysis (Corbin \& Strauss, 2008), while the survey data were used to test and refine concepts and categories (Creswell, 2009). Once open codes were generated through the microanalysis, they were subjected to axial coding to combine similar codes (Corbin \& Strauss, 2008). At this point, clear concepts began to emerge, which were then applied to the survey data and adjusted where necessary (Corbin \& Strauss, 2008). The concepts were developed and refined against the two sources of data until the researcher and auditors agreed that saturation had been achieved. At this point, the concepts seemed to apply to one of several categories, and were grouped accordingly. Specifically, some concepts spoke to the IP experience, others referred to contextual factors stimulating or inhibiting imposter feelings, while others described various outcomes resulting from the IP. Once these categories were developed, they were reexamined against the survey and interview data, audited, and refined until saturation seemed to be achieved. At this point, the interviewees 
were invited to examine the concepts and categories and provide feedback (Creswell, 2009).

Two interviewees accepted this invitation, and both agreed that the findings were applicable and salient to their experiences.

\section{Trustworthiness of the Data}

Various methods were utilized to assure trustworthiness and rigor (Creswell, 2009; Kline, 2008). These included consultation with colleagues throughout the design and analysis processes, analyzing multiple sources of data, analysis auditing using multiple auditors, and participant checks of the concepts and categories. These measures maximized the likelihood of credible analysis and minimized the possibility of misinterpretation of the findings (Creswell, 2009). Throughout this process, an audit trail was maintained in the interest of transparency (Kline, 2008).

\section{Results}

Of the 29 participants, an unexpectedly high number $(n=23,79.3 \%)$ indicated that they experience imposter feelings, and most offered specific examples of experiences consistent with the IP. All nine of the male participants, and 14 of the female participants $(70.0 \%)$, identified with the IP. In examining questionnaire and interview responses using the aforementioned analytic procedures, clear categories and concepts emerged. These concepts described the IP experience, identified internal and external factors that either promoted or inhibited imposter feelings, and described how the IP engendered both performance and affective reactions. These concepts constituted the grounded theory model, summarized below. Participant quotations come from both the interview and survey data. For each category, the concepts are reported in order from most to least commonly occurring in the data.

\section{The Imposter Experience}


The first - and most commonly described - category pertained to the actual IP experience. Participants consistently recounted IP experiences in both academic and professional settings. Despite the range of backgrounds among these participants, many commonalities existed. The participant IP experiences were consistent with descriptions from prior literature; they included perceived fraudulence, discrediting evidence of competence, and self-doubt.

Perceived fraudulence. Many participants relayed a sense of phoniness related to their abilities or accomplishments. These perceptions were most common when acclimating to new levels of academic or professional responsibility, but they also occurred in other situations. Often, participants talked of believing that others would eventually discover their phoniness. A 25-year-old male who had earned a Bachelor's degree in Museum Studies but was working in retail summarized this sentiment: "In college classes I was paranoid that every paper I wrote would be exposed as [a] complete lack of knowledge and seen as what a 'hack' student would write".

Discrediting evidence of competence. The data yielded many examples of individuals minimizing achievements or praise they had received. Repeatedly, participants conveyed disbelief that their skills or abilities contributed to hirings, promotions, grades, and even their undergraduate degrees. Rather, they attributed such accomplishments to luck, good fortune, or some other external cause. Interestingly, the pervasiveness of their discrediting was such that, when receiving external validation regarding their accomplishments, many participants questioned the motives or faculties of the person offering praise. Such was the case with a 25year-old male professional musician:

Sometimes, I get these superfluous compliments, and I feel like the [person giving the 
compliment] just has it all wrong. The other day, I played a show that I could barely get through on the trumpet. Every note was on the verge of cracking. The next day, a professional Jazz trumpet player posted on my [Facebook] wall that he loved my tone, which was a joke to me because I just got through [the performance] by the skin of my teeth.

Self-doubt. Participants also commonly believed they lacked qualifications or abilities. Self-doubt was often described in anticipation, such as when individuals had applied for jobs or graduate school acceptance and were awaiting responses. Often, participants expressed awareness that they possessed impressive qualifications, but were nevertheless susceptible to self-doubt. This paradoxical thinking was clearly present in the response of a 23 -year-old female who worked at a document scanning company but was preparing to enter law school: "I scored in the 99th percentile on the LSAT, got into a top school with a sizable scholarship, and I still worry that I will do poorly and not get a job". Other times, however, self-doubt was described more broadly. In these cases, participants reported a general sense that they lacked confidence or were low in self-worth. One participant succinctly yet powerfully summarized this feeling: "One of my biggest fears in life is that I'm not good enough".

\section{Internal and External Factors Impacting the IP}

Frequently, participants attributed their imposter feelings to specific character traits. They also described external behaviors and situations that either promoted or inhibited the IP. Thus, a character profile emerged suggesting that emerging adults who are highly motivated and perfectionistic, and who have difficulty self-validating, may be especially prone to the IP. External factors that contributed to the IP included instances in which the participants compared themselves to others or received evaluative feedback; however, gaining experience was an 
external factor that participants felt diminished the IP.

Internal qualities. Several domains of internal qualities emerged that seemed to directly contribute to the IP, including high motivation and perfectionism, as well as an inability to selfvalidate. A response from one participant summarized the interrelatedness of these qualities: "I don't always feel okay with myself in terms of accepting the good; I usually look at the negative and how I can improve, and I don't pat myself on the back with the positives”.

Participants tended to be highly achievement-focused and to hold themselves to lofty standards. Many reported continually working on self-improvement and maximizing their potential. Some indicated a perception of relatedness between motivation and imposter feelings. One participant, who said her imposter feelings began recently, differentiated between feeling like an imposter presently and having less motivation in the past:

I don't think I had these feelings back when I was younger. I don't think I really did anything to try to achieve like I am now. [...] I think I realized as I got older it's more than just getting good grades. You have to, um...be able to network, and uh...you know, just because you have a 4.0 in school doesn't mean that you're going to get the job. Um...you need experience and stuff like that.

Participants also expressed a need for external praise in order to feel confident. Some were able to identify their inability to self-validate, while others lacked this insight. Regardless, the majority, when asked about their sources of confidence, described only external factors, such as receiving a compliment or a good grade. One male participant, while considering his employment experiences, concluded:

I've always second-guessed if what I was doing was right. Always kind of relying on my boss to make the final say. I've never kind of, I don't know, owned what I have to do, 
unless it was a clear, easy decision to make. So, I guess, personally my confidence level has always been a little shaky just because I wasn't sure if I was [right and] I wasn't getting the affirmation I think I needed.

The fact that many participants looked to others for validation was noteworthy given the aforementioned category of discrediting evidence of competence. That is, participants expressed a desire for external validation and an inability to provide it for themselves. Yet, when they received this validation, they discredited it, often going so far as to question the motives of the validator.

External factors. Though participants relied upon external sources for validation, feedback from others also seemed to impact imposter feelings in several ways. Participants repeatedly described comparing their performance or perceived abilities with others. They also reported that evaluative periods at work were instances in which imposter feelings were elevated. The external factor of gaining experience, however, contributed to diminished imposter feelings. The theme of comparison was salient among participants. Various types of comparative behaviors were described, all of which seemed to stimulate imposter feelings. Opportunities for comparison included witnessing peers succeed, interacting with colleagues on group projects at work, and speculating about the confidence of others. The relationship between comparison and the IP was multifaceted. The most common description of comparison involved ways that it increased imposter feelings. Such feelings were stimulated when participants perceived that others were successful or capable. Participants also speculated about the confidence of others, believing others to possess significantly more confidence than themselves. This theme was accurately reflected by a female participant preparing to graduate from a business administration honors program: "I always feel unsure when comparing myself to others, everyone around me 
seems so confident and further along on the path than me".

Conversely, observing colleagues struggle with work tasks seemed to promote confidence and normalize developmental areas. One participant, a 25-year-old counselor, described her experience witnessing colleagues struggle during group supervision: "Seeing others struggle I've realized that I'm kind of with them. It's like a 'together' kind of struggle, so I don't feel like I'm worse than a lot of people [...] I just feel like we're all together'. Equally salient was the reported sense of relief that nearly every one of these individuals described as a result of participating in the present study and learning that others experienced the IP as well. One participant's response summarized this sentiment:

It is very relieving to find out about the imposter phenomenon. I never questioned that my experience was genuine, but if other people feel this very specific experience as well, that opens the possibility that it is in my head.

In addition to comparison, the IP was influenced in different ways by receiving evaluations. Many spoke of instances in which in-person evaluations of work or academic performance resulted in particularly strong imposter feelings, even if the evaluation was generally positive. One participant relayed an experience in which she was shocked to receive a glowing evaluation during a quarterly evaluation at her sales position; she had been considering quitting the job as the evaluation approached because she had been "certain" that her performance would be negatively evaluated and that she would be demoted. For others, positive evaluations represented an opportunity to receive external validation, resulting in feeling competent.

A final external factor impacting the IP was experience. Some participants felt that, as they gained more professional experience, their imposter feelings became less frequent and 
pervasive. This was particularly true when the experience allowed them to feel prepared for future experiences. Conversely, more challenging tasks promoted significant imposter feelings. Participants who felt they lacked experience also believed that gaining experience would help them feel competent, as was indicated by a recent college graduate: "Right now I feel inadequate, but it's probably just because I don't really have too much experience".

\section{The Impact of the Imposter Phenomenon}

The IP impacted emerging adults both internally and externally. A few participants believed that the effects of the IP were solely internal, as was the case with one male participant, who speculated that if one was “watching me work I don't think you would have seen any difference in how I was working". The majority of participants, however, reported that their imposter beliefs impacted both performance and affect.

Performance impact. Participants pointed to a range of performance reactions related to the IP. These reactions included both performance benefits and deficits. The primary performance benefit was increased motivation to work hard and feel competent. One participant, who reported that she first felt the IP near the end of her undergraduate degree program, recalled:

I realized that I was going to be graduating and I didn't really have anything to put on my résumé other than school, so that was when I really started scrambling and finding internships and jobs and joining clubs, and stuff like that.

Other participants, however, reported that the IP negatively impacted their performance.

The most common way in which performance was inhibited was through avoidance. Participants identified several types of avoidant behaviors in which they engaged, seemingly as an effort to ignore their perceived incompetence. Such avoidant behaviors ranged from small activities, such as procrastination or not asking questions in class for fear of sounding unintelligent, to making 
major life decisions based on assumptions of limited capability. Such was the case with a male college graduate struggling to find a job, saying that the "professional world has shunned me based upon my choice to go to a 'not so well known' university. [...] I chose this university based on fear that I would be exposed at a major university".

Various verbal and nonverbal nervous habits represented another type of performance deficit. The most common nervous behavior pertained to verbal communication with colleagues, superiors, or prospective employers. Participants described many instances of "stumbling over their words" and a few instances of feeling like they were misrepresenting themselves while at job interviews, meetings, or classes. A few participants also reported various nonverbal behaviors they felt betrayed their internal feeling of inadequacy, such as nervous tics or staring at their feet.

Affective impact. Participants also conveyed a sense that the IP resulted in various affective responses. The most common of these was the experience of fear, worry, or anxiety. For some, anxiety was a general feeling in response to imposter experiences, but others reported specific fears that they would be fired, demoted, or that they would fail an assignment or class. A 24-year-old male middle school teacher explained, "I often consider myself unworthy of my job and worry it is only a matter of time before they find someone more qualified to replace me". Some participants described a temporary sense of relief accompanying the completion of IPinducing tasks or evaluations, though their anxiety would return when they began thinking about new tasks or future evaluations.

In addition to anxiety, many participants described how the discrediting aspects of the imposter experience resulted in their inability to build upon achievements, abilities, or external validation. One participant described a pattern of second-guessing that would occur in response 
to compliments; this pattern would continue until "it was as if no one had given me a compliment anyway. Like, the net effect was missing”. Another participant attempted to articulate the disconnect between logically recognizing her abilities and allowing them to help her feel competent:

I know [my strengths are] there, I just don't bring them in. You know, like if someone asked me to say my strengths or whatever, I can write them down, but I just...the internalizing part is where...So like, they're there, and I know they're there, I just don't know they're there (laughs).

A less common but particularly salient affective reaction for a few participants was the feeling of guilt in response to accolades or achievements. Participants reported guilt when receiving praise for achievements they felt were unearned or when outperforming peers or colleagues. One participant, a female graduate student, seemed to provide an example of this concept when asked about the cause of her imposter feelings:

It's been $[\ldots]$ within the past $[\ldots]$ four or five years. [...] As I've advanced farther, and you know, seen some friends that kind of stayed behind, you know, stuck in high school days or something like that. And I feel like I'm advancing and they're not.

\section{Discussion}

The present study provided a model for understanding the IP and its experience in emerging adulthood. Sequential differences were found in specific imposter experiences: 1) selfdoubt was an anticipatory experience, 2) perceived fraudulence was experienced in the midst of completing a task, and 3) discrediting was a reflective experience. These themes add complexity to current knowledge and provide confirmation of existing quantitative IP research, notably the demonstrated link between the IP and low self-esteem (Sonnak \& Towell, 2001). The 
descriptions of participant IP experiences also demonstrate the continued applicability of the construct proposed over 30 years ago (Clance \& Imes, 1978).

Internal qualities, such as perfectionism and an inability to self-validate, seemed to promote imposter feelings. So too did the external experiences of comparison and evaluation. Gaining experience, however, contributed to diminished imposter feelings, at least with regard to the task for which experience was gained. This finding contrasts that of the Royse-Roskowski (2010) study, which found no relationship between the IP and experience. Furthermore, the findings suggest that evaluative periods promote imposter feelings. This finding echoes those of a study conducted by Thompson, Foreman, and Martin (2000), which suggested that much of the anxiety caused by the IP relates to the possibility of being negatively evaluated. This anxiety might relate to the aforementioned inability to self-validate. In tandem, the findings suggest that needing external validation may actually interfere with evaluation periods, a time in which potential exists to receive external validation.

The IP impacted the sample externally by contributing to both productive (i.e., increased effort and motivation) and counterproductive (i.e., avoidant and nervous behaviors) behavioral reactions. These themes support previous quantitative findings linking the IP to various performance benefits (Caselman et al., 2006) and deficits (Cowman \& Ferrari, 2002; Want \& Kleitman, 2006). Caselman et al. (2006) argued that the enhanced motivation that can result from the IP might also perpetuate its experience. An individual who compensates for feeling incompetent by overworking, for example, could inadvertently validate a system attributing subsequent success to the extra effort exerted and not to internal abilities. Such a system reinforces a message that "I am a fraud, and the only way to match the productivity of my more capable peers is to work harder than them". 
Finally, the findings suggested various affective reactions to the IP, including anxiety, an inability to internalize success, and, at times, guilt. These reactions share interesting similarities with Bowlby's (1969/1982) attachment theory, specifically with an anxious attachment style. In theory, an anxious attachment style is associated with negative beliefs regarding the self and positive beliefs regarding the usefulness and abilities of others, resulting in feelings of inadequacy and maladaptive interpersonal dependence. A recent study of female graduate students identified a relationship between imposter feelings and anxious attachment (GibsonBeverly \& Schwartz, 2008). In the context of the present study, the consistency of the findings to an anxious attachment pattern is noteworthy given attachment theory's contention that insecure attachment strategies become activated in times of elevated distress (Bowlby, 1969/1982). It is possible that the initial transition to worklife may be particularly distressing for emerging adults (Lane, 2014). Further research is needed to better understand the affective experience of this normative emerging adult life transition.

An interesting aspect of these findings is the mechanism they elucidate by which praise is received and processed. The findings suggest that emerging adults experiencing the IP are caught in a cycle in which they require external sources of validation (e.g. praise, comparisons to the performance of others) to feel competent, and yet, when they receive such validation, engage in discrediting behaviors that prevent them from internalizing it. This cycle suggests that the benefits of external validation are temporary and do little to promote long-term self-efficacy among emerging adults experiencing the IP.

Also of interest was the consistent sentiment that participating in the study and learning about the IP was relieving for participants. By discovering that others experienced imposter feelings, participants learned they were not alone in their fears, and many began to consider 
whether or not their feelings were founded. This finding suggests that normalizing the IP could constitute an important aspect of its treatment, which supports the recommendation of Clance and Imes (1978) that group counseling represents an effective treatment approach.

One other interesting finding was the unexpected prevalence of imposter feelings among participants. That nearly $80 \%$ of the participants identified with the IP is surprising given other prevalence estimates. Sonnak and Towell (2001), for example, reported that $43 \%$ of their sample of University students met the cutoff score requirements for the IP. The conclusions that can be drawn from this finding are limited, however, as the present study was not designed to examine the prevalence of the IP in emerging adulthood with any degree of rigor. Nevertheless, the finding was noteworthy and suggests a future research direction.

\section{Limitations}

Though steps were taken to ensure credibility, dependability, and objectivity, this study is not without its limitations. The sample was predominantly Caucasian and female. The disproportionate number of female participants is noteworthy given that many have argued that females are more susceptible to the IP than males (Clance \& Imes, 1978; Gibson-Beverly \& Schwartz, 2008). This assertion, however, has been disputed by other studies that have reported no significant gender differences (Fried-Buchalter, 1997; Royse-Roskoski, 2010). Nonetheless, a more representative sample would strengthen the findings.

\section{Practical Applications}

Despite these limitations, the findings provide potentially useful contributions for counselors working with emerging adults. The IP is an internal experience and is not a common presenting issue (Gibson-Beverly \& Schwartz, 2008); however, imposter feelings may contribute to, interact with, or result from other emotional difficulties (Clance \& O'Toole, 1988). The 
present findings, then, are useful to counselors in that they elucidate the IP experience as well as its antecedents and consequences. Thus, counselors may be better equipped to recognize IP experiences in their clients and, in turn, actively address them.

Additionally, the findings suggest two concepts of possible relevance to treating the IP for transitioning emerging adults. First, the aforementioned cycle related to validation emphasizes the importance that emerging adults develop an ability to self-validate. That is, it seemed common for the sample to have difficulties internalizing positive beliefs about themselves and their work, and so they sought validation from others. This validation, however, seemed to provide only temporary relief, perpetuating the cycle of seeking additional validation. That some emerging adults transitioning into professional settings would have difficulties selfvalidating is, perhaps, not surprising, since beginning work life often corresponds with decreased proximity to familial, peer, and institutional support networks (Lane, 2013). Therefore, interventions should be sought that encourage development of self-validation skills. It is possible that approaches such as dialectical behavior therapy (Linehan, 1993) or mindfulness (Rogers \& Maytan, 2012) could represent ideal modalities given their emphasis on teaching clients to develop self-awareness regarding emotional processes that perpetuate problematic behavioral reactions. Such modalities could teach emerging adult clients that their desire to feel competent is healthy and appropriate, but the behaviors they choose to satiate those desires (i.e., seeking external validation) are not providing them the enduring relief they seek.

Second, a common sentiment among participants was that learning about the IP through the surveys and interviews was relieving. It is possible that normalizing IP experiences can aid the treatment process for emerging adult clients. Clance and Imes (1978) first articulated this idea, suggesting group therapy as an effective intervention for individuals with imposter feelings. 
The group context promotes universality and vicarious learning (Lane, 2013). By discovering that other emerging adults experience imposter feelings while they transition into career settings, perhaps clients would be encouraged to consider a developmental perspective regarding their own imposter feelings, in which such feelings are a relatively common experience during this transition. Such a perspective would likely help reduce the power of imposter narratives.

\section{Implications for Future Research}

This study examined the IP in emerging adults transitioning into professional life. Its findings suggest that further research in this area would be useful. Primarily, the findings underscore the importance of adding to the presently sparse literature examining the IP in emerging adulthood. Much of the existing IP research was conducted during the 1980's, a time that predates many of the societal shifts that have influenced today's 18 - to 25 -year-olds. Moreover, future research should quantitatively test the grounded model and examine the generalizability of the model with other populations. Additional qualitative research seeking to understand how the experience of the IP changes over time would also be of use. Finally, given that $80 \%$ of the sample identified with the IP, quantitatively examining its prevalence among emerging adults seems warranted. These studies would aid researchers and clinicians in better understanding the degree to which emerging adult life transitions stimulate the IP. 


\section{References}

Al-Darmaki, F. R. (2004). Counselor training, anxiety, and counseling self-efficacy: Implications for training psychology students from the United Arab Emirates University. Social Behavior \& Personality: An International Journey, 32(5), 429-439.

Allen, J. K., \& Taylor, K. (2006). The senior year transition: Women undergraduates search for a path. Journal of College Student Development, 47(6), 595-608.

Arnett, J. J. (2000). Emerging adulthood: A theory of development from the late teens through the twenties. American Psychologist, 55(5), 469-480.

Arnett, J. J. (2001). Conceptions of the transition to adulthood: Perspectives from adolescence through midlife. Journal of Adult Development, 8(2), 133-143.

Bell, B. S., \& Kozlowski, S. W. J. (2002). Goal orientation and ability: Interactive effects on self-efficacy, performance, and knowledge. Journal of Applied Psychology, 87(3), 497505.

Bernard, N. S., Dollinger, S. J., \& Ramaniah, N. V. (2002). Applying the big five personality factors to the impostor phenomenon. Journal of Personality Assessment, 78(2), 321-333.

Bowlby, J. (1969/1982). Attachment and loss: Vol. 1. Attachment. New York: Basic Books.

Caselman, T. D., Self, P. A., \& Self, A. L. (2006). Adolescent attributes contributing to the imposter phenomenon. Journal of Adolescence, 29, 395-405.

Cherniss, C. (1980). Professional burnout in human service organizations. New York: Praeger.

Clance, P. R., \& Imes, S. A. (1978). The imposter phenomenon among high achieving women: Dynamics and therapeutic intervention. Psychotherapy Theory, Research, and Practice, $15(3), 241-247$.

Clance, P. R., \& O’Toole, M. A. (1988). The imposter phenomenon: An internal barrier to empowerment and achievement. Women and Therapy, 6, 51-64. 
Clark, Vardeman, \& Barba (in press). Perceived inadequacy: A study of the imposter phenomenon among college and research librarians. College \& Research Libraries. Retrieved from http://crl.acrl.org/content/early/2012/12/19/crl12-423.short

Corbin, J., \& Strauss, A. (2008). Basics of qualitative research: Techniques and procedures for developing grounded theory ( $3^{\text {rd }}$ ed.). Los Angeles, CA: Sage.

Cowman, S. E., \& Ferrari, J. R. (2002). “Am I for real?” Predicting impostor tendencies from self-handicapping and affective components. Social Behavior and Personality, 30(2), $119-125$.

Creswell, J. W. (2009). Research design: Qualitative, quantitative, and mixed methods approaches. Los Angeles, CA: Sage.

Denzin, N. K. (2006). Sociological methods: A sourcebook $\left(5^{\text {th }}\right.$ ed.). Piscataway, NJ: Transaction Publishers.

Fried-Buchalter, S. (1997). Fear of success, fear of failure, and the imposter phenomenon among male and female marketing managers. Sex Roles, 37(11/12), 847-859.

Gibson-Beverly, G., \& Schwartz, J. P. (2008). Attachment, entitlement, and the impostor phenomenon in female graduate students. Journal of College Counseling, 11, 119-132.

Judge, T. A., Jackson, C. L., Shaw, J. C., Scott, B. A., \& Rich, B. L. (2007). Self-efficacy and work-related performance: The integral role of individual differences. Journal of Applied Psychology, 92(1), 107-127.

Kline, W. B. (2008). Developing and submitting credible qualitative manuscripts. Journal of Counselor Education and Supervision, 47(4), 210-217.

Kolligian, J., Jr., \& Sternberg, R. J. (1991). Perceived fraudulence in young adults: Is there an “imposter syndrome”? Journal of Personality Assessment, 56(2), 308-326. 
Kruger, D. J., Fisher, M. L., Strout, S. L., Wehbe, M., Lewis, S., \& Clark, S. (2013). Variation in women's mating strategies depicted in the works and words of Jane Austen. Journal of Social, Evolutionary, and Cultural Psychology, 7(3), 197-210.

Lane, J. A. (2013). Group counseling for students transitioning out of postsecondary education: A narrative approach. Groupwork: An Interdisciplinary Journal for Working With Groups, 23(1), 34-55. doi:10.1921/1501230103

Lane, J. A. (2014). Attachment, social support, and well-being among emerging adults in transition. Manuscript submitted for publication.

Lane, J. A. (in press). Attachment, well-being, and college senior attitudes about the transition out of college. Journal of College Counseling.

Leary, M. R., Patton, K. M., Orlando, A. E., \& Funk, W. W. (2000). The impostor phenomenon: Self-reflections, reflected appraisals, and interpersonal strategies. Journal of Personality, 68(4), 725-756.

Linehan, M. M. (1993). Cognitive-behavior treatment of borderline personality disorder. New York: Guilford.

McGregor, L. N., Gee, D. E., \& Posey, K. E. (2008). I feel like a fraud and it depresses me: The relation between the imposter phenomenon and depression. Social Behavior and Personality, 36(1), 43-48.

Murphy, K. A., Blustein, D. L., Bohlig, A. J., \& Platt, M. G. (2010). The college-to-career transition: An exploration of emerging adulthood. Journal of Counseling \& Development, 88(2), 174-181.

Padilla-Walker, L. M., \& Nelson, L. J. (2012). Black hawk down?: Establishing helicopter parenting as a distinct construct from other forms of parental control during emerging 
adulthood. Journal of Adolescence, 35(5), 1177-1190.

Polach, J. L. (2004) Understanding the experience of college graduates during their first year of employment. Human Resource Development Quarterly, 15, 5-23.

Rogers, H., \& Maytan, M. (2012). Mindfulness for the next generation: Helping emerging adults manage stress and lead healthier lives. New York: Oxford.

Royse-Roskowski, J. C. (2010). Imposter phenomenon and counseling self-efficacy: The impact of imposter feelings (Doctoral dissertation, Ball State University). Retrieved from http://cardinalscholar.bsu.edu/handle/123456789/194625

Saks, A. M., Uggerslev, K. L., \& Fassina, N. E. (2007). Socialization tactics and newcomer adjustment: A meta-analytic review and test of a model. Journal of Vocational Behavior, 70(3), 413-446.

Schnyders, C., \& Lane, J. A. (2014). Gender differences regarding parental and peer attachments in early emerging adulthood experiences. Manuscript submitted for publication.

Sonnak, C., \& Towell, T. (2001). The impostor phenomenon in British university students: Relationships between self-esteem, mental health, parental rearing style and socioeconomic status. Personality and Individual Differences, 31, 863-874.

Thompson, T., Foreman, P., \& Martin, F. (2000). Imposter fears and perfectionistic concerns over mistakes. Personality and Individual Differences, 29, 629-647.

Topping, M. E. H. (1983). The impostor phenomenon: A study of its construct validity and incidence in university faculty members. Dissertation Abstracts International, 44, 1948B-1949B.

Twenge, J. M. (2013). The evidence for Generation Me and against Generation We. Emerging 
Adulthood, 1(1), 11-16.

Want, J., \& Kleitman, S. (2006). Imposter phenomenon and self-handicapping: Links with parenting styles and self-confidence. Personality and Individual Differences, 40, 961-97. 